\title{
Comparing Single-Objective Optimization Protocols for Calibrating the Birds Nest Aquifer Model-A Problem Having Multiple Local Optima
}

\author{
Richard T. Lyons ${ }^{1}$, Richard C. Peralta ${ }^{1, *}$ and Partha Majumder ${ }^{2}$ \\ 1 Department of Civil and Environmental Engineering, Utah State University, Logan, UT 84322-4110, USA; \\ ricklyons@mac.com \\ 2 College of Water Conservancy and Hydropower Engineering, Hohai University, Nanjing 211100, Jiangsu, \\ China; parthamajp@gmail.com \\ * Correspondence: peralta.rc@gmail.com; Tel.: +1-435-881-4947
}

Received: 1 January 2020; Accepted: 27 January 2020; Published: 30 January 2020

check for updates

\begin{abstract}
To best represent reality, simulation models of environmental and health-related systems might be very nonlinear. Model calibration ideally identifies globally optimal sets of parameters to use for subsequent prediction. For a nonlinear system having multiple local optima, calibration can be tedious. For such a system, we contrast calibration results from PEST, a commonly used automated parameter estimation program versus several meta-heuristic global optimizers available as external packages for the Python computer language — the Gray Wolf Optimization (GWO) algorithm; the DYCORS optimizer framework with a Radial Basis Function surrogate simulator (DRB); and particle swarm optimization (PSO). We ran each optimizer 15 times, with nearly 10,000 MODFLOW simulations per run for the global optimizers, to calibrate a steady-state, groundwater flow simulation model of the complex Birds Nest aquifer, a three-layer system having 8 horizontal hydraulic conductivity zones and 25 head observation locations. In calibrating the eight hydraulic conductivity values, GWO averaged the best root mean squared error (RMSE) between observed and simulated heads-20 percent better (lower) than the next lowest optimizer, DRB. The best PEST run matched the best GWO RMSE, but both the average PEST RMSE and the range of PEST RMSE results were an order of magnitude larger than any of the global optimizers.
\end{abstract}

Keywords: calibration; optimization; nonlinear optimization; groundwater; Grey Wolf Optimization; Particle Swarm Optimization; Meta-Heuristic Optimization; PEST calibration; Birds Nest Aquifer; Uinta Basin

\section{Introduction}

Groundwater simulation models help engineers and scientists better understand water flow systems and potential effects of pumping and other diversions introduced into real-world systems. Model calibration is an essential step in demonstrating that a model can appropriately represent the real world. The process of calibrating a contaminant transport models first involves calibrating a flow model. PEST has been used extensively to reduce the effort and improve the quality of model calibrations [1,2]. PEST and PEST++ are available as a stand-alone command-line run computer program and some version of PEST is also integrated within several MODFLOW-based groundwater modeling software packages—Visual MODFLOW [3], Groundwater Vistas [4], Processing Modflow [5], and Groundwater Modeling System (GMS) [6].

Simulation model calibration can be viewed as an optimization problem. Global optimization techniques have long been considered and used for groundwater calibration $[7,8]$. The calibration goal is to determine the set of decision variable values that enable the model to produce output 
hydrologic estimates that best match values observed in the field- to produce the smallest root mean squared error (RMSE) between simulated and observed values. PEST uses the gradient search-based Gauss-Marquardt-Levenberg (GML) method to identify the set of decision variables that are the best parameter value inputs to the simulation model.

The GML method works well for identifying a locally optimal solution for a convex region of the solution space. However, the GML method cannot guarantee finding the globally optimal solution for a nonlinear solution space that has multiple local optima. [9-11].

To address the situation of multiple optima in preliminary modeling efforts, we made multiple runs, beginning each with a different initial guess of the optimal solution. Each initial guess converged to the nearest optimal solution. Comparison of the RMSEs of the different locally optimal solutions enabled selecting a solution that provided the lowest RMSE as a globally optimal solution. A challenge to this approach is that a user does not initially know how many optimal solutions exist. A PEST user can somewhat address this by making an exhaustive set of runs that use all practical combinations of maximum and minimum parameter values. As the number of parameters being calibrated increases, the modeler becomes less confident that they have identified all the most important optima. Because the global optimizers in this study randomly choose their starting input variables, instead of using an exhaustive set of runs, we created 15 sets of 8 randomly selected input parameters as our PEST initial values.

PEST is the de facto option for groundwater calibration, and is most commonly used. We hypothesized that optimization algorithms developed specially to address very nonlinear systems could be useful for calibration. Their use could potentially also decrease the complexity of calibration preparation. Because meta-heuristic optimization techniques avoid entrapment in local optima [12], here we evaluate use of several meta-heuristic algorithms for calibration.

Some recent groundwater studies suggest using newer algorithms for groundwater calibration and parameter estimation-Ensemble Kalman filtering [13]; null-space Monte Carlo [14]; Particle Swarm Optimization (PSO); Pattern Search [15]; Differential Evolution, Cat-Swarm Optimizer (CSO), and Particle Swarm Optimizer(PSO) [16]; CSO, PSO, and Grey Wolf Optimizer (GWO) [17].

The meta-heuristic PSO [18] introduced an innovative optimization method based on nature-based bird flocking and swarming theory. PSO has been used extensively in water resources engineering [19-21], and even as a calibration tool [15].

A newer swarm-based GWO furthers nature-based optimization by introducing a scheme based on the social hierarchy and hunting behavior of grey wolves. Compared with other swarm-based optimizers such as PSO, GWO has fewer adjustable parameters and allows wolves/search agents to approach, encircle and attack prey/optima [12]. GWO has been quickly adapted and used in many fields including water engineering, but has been used little in groundwater modeling $[17,22]$.

Response surface methods of expressing an objective function value, such as radial basis function interpolation, are flexible, do not require derivatives, and can avoid entrapment in local optima, but are not as simple to implement as some meta-heuristic methods. Radial basis function interpolation is a well-known, mesh-free, response surface model [23] and has been used in groundwater engineering [24]. The DYCORS optimization framework was developed to use radial basis functions in highly-dimensional, black-box processes. The DYCORS/radial basis function (DRB) uses a dynamic search strategy that perturbs only a subset of the current best solution until an optimal solution is found [25].

The discussed PSO, GWO, and DRB optimizers are available as third-party black-box optimizer packages with the Python computer language. A high-level, easy to learn language, Python is easily expanded by third-party packages that are not included in the base Python language. This feature allows ready access to pre-built optimizers that are beyond the scope of the general Python writers and maintainers. It allows engineers and scientists to access and use codes that might otherwise be difficult and time-consuming to reproduce. 
In this calibration comparison we use PSO, GWO, and DRB optimizers through Python, and compare results with the results from PEST. Although these meta-heuristic optimization algorithms are not new, their easy implementation through Python can allow them to effectively complement PEST use. Comparing results might encourage use of the optimizers for calibrating models of very nonlinear systems, especially groundwater systems.

\section{Materials and Methods}

The goal of this study is to compare the use of three metaheuristic optimizers versus PEST for calibrating horizontal hydraulic conductivity values for a Birds Nest Aquifer (BNA) simulation model. In collaboration with the State of Utah, Division of Oil, Gas, and Mining, this study is a step toward future modeling that will evaluate horizontal flow patterns resulting from potential water injection. Calibration runs to date employed a personal computer with Windows 10 operating system. We ran PEST using the command line interface. Preliminary PEST tests involving changing the PEST default options did not noticeably affect processing time or the computed RMSE. Therefore, all PEST runs subsequently reported here used PEST default options.

We ran PSO, GWO, and DRB in Python using third-party packages-Pyswarm [26], SwarmPackagePy [27], and pySOT [23]. We used PSO by utilizing the default options of Pyswarm. We implemented GWO through SwarmPackagePy. We employed DRB through PySOT (Python Surrogate Optimization Toolbox). Of PySOT's options, we used the radial basis function surrogate model (default), Symmetric Latin hypercube experimental design, and DYCORS search strategy [25].

Table 1 illustrates that as general input, the above global optimizers require the number of dimensions (number of decision variables to be optimized), upper and lower bounds on decision variables, maximum number of allowable iterations, and number of optimizer agents. Each optimizer uses a different equation for the total number of MODFLOW simulations to run, based on agent size and number of iterations. The total number of GWO simulations equals the number of agents times the number of iterations plus two times the number of agents plus two times the number of iterations. So, 30 agents and 300 iterations yields $30 \times 300+2(30)+2(300)=9660$. Total number of PSO simulations equals the number of agents times the number of iterations plus the number of agents. So, 30 agents and 321 iterations yields $30 \times 321+30=9660$. Total number of DRB simulations is merely the number of iterations. An agent is one set of candidate solutions, consisting of all decision variables. So, for a model with 8 dimensions, an optimizer using 30 agents would use 30 sets, each having 8 decision variables. GWO automatically performs the full number of iterations and therefore does not require any further inputs. We used default values for the additional inputs that PSO requires: particle velocity scaling factor (0.5), scaling factors to control search away from the best positions of each particle and the swarm $(0.5,0.5)$, minimum step size before search termination $\left(1 \times 10^{-8}\right)$, and minimum change of swarm's best objective value before search termination $\left(1 \times 10^{-8}\right)$. We used default values for the additional inputs required by the DRB surrogate model: a kernel object (CubicKernel), a polynomial tail object (LinearTail(8)), and a regularization parameter $\left(1 \times 10^{-6}\right)$.

We coded a Python script to run our calibration. The script loads the appropriate optimizer inputs into each optimizer. The optimizer creates a group of decision variables that the Python script writes to a MODFLOW input file. The script then instructs MODFLOW to run, producing an output file of well heads. The Python-scripted objective function file reads the well heads and calculates a fitness value that is read by the optimizer. This process continues until the maximum number of iterations are run or a search termination criterion is satisfied. The optimizer then reports the decision variables and the fitness value of the best result. The process is shown in Figure 1. 
Table 1. The three global optimizers, their respective Python packages, common inputs needed and additional inputs. PSO: Particle Swarm Optimization; GWO: Gray Wolf Optimization; DRB: DYCORS/radial basis function.

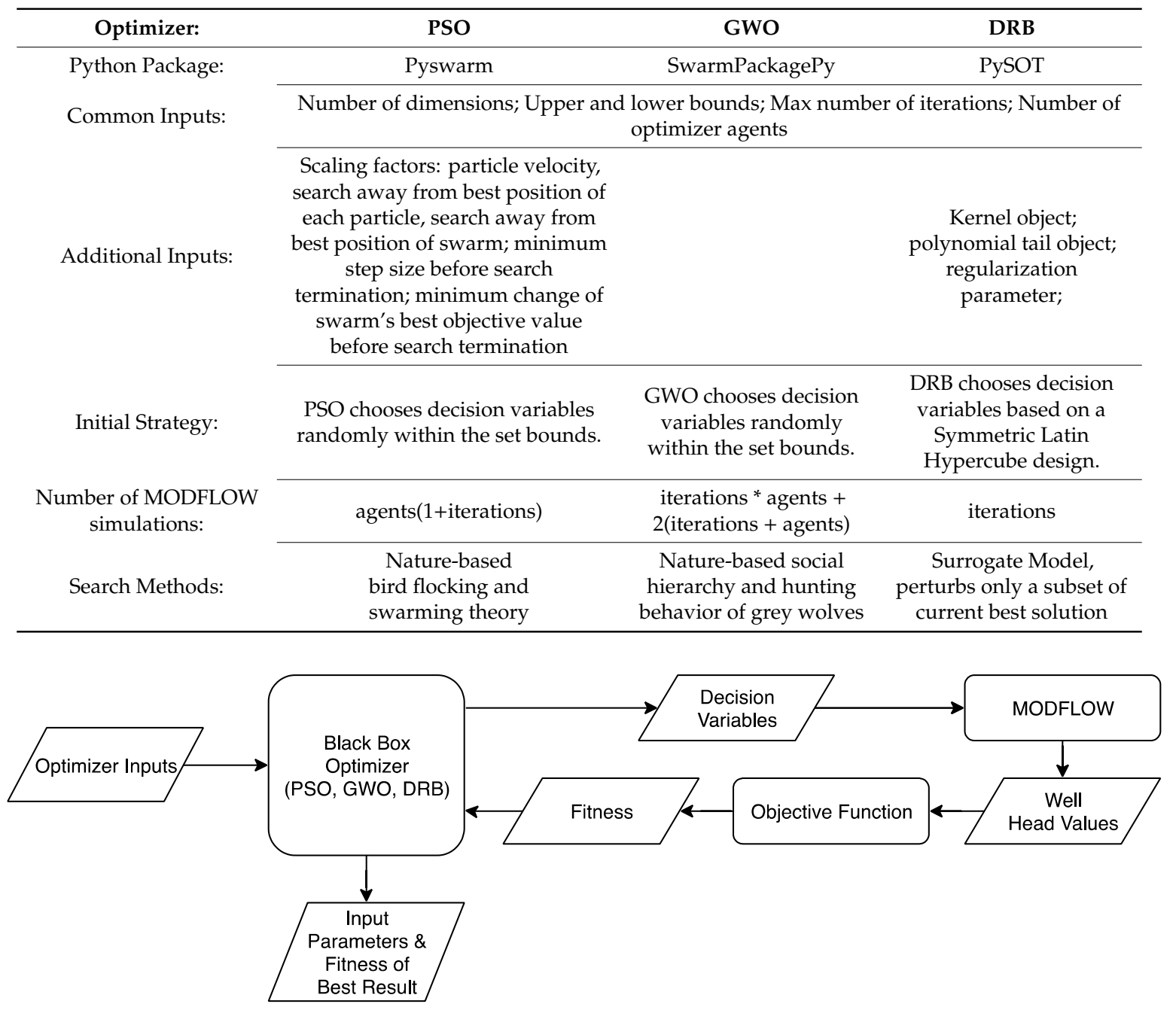

Figure 1. Flowchart of calibration process for the global optimizers. PSO: Particle Swarm Optimization; GWO: Gray Wolf Optimization; DRB: DYCORS/radial basis function.

Site

The roughly 700 square mile BNA (Figure 2), is located in the Uinta Basin in north-eastern Utah. This Eocene era aquifer lies beneath the unconfined Uintah and Duchesne aquifers. BNA base lies at depths beneath the ground surface ranging from about 210 feet to nearly 5800 feet. The BNA is recharged primarily by assumedly uniform seepage from the Uinta aquifer, and by seepage mainly from Evacuation Creek in the south east [28]. Discharge, mainly via a spring near Bitter Creek, follows a path probably facilitated by a Gilsonite vein [28]. The three-layers of the aquifer consist mainly of organic-lean oil shale. The top and bottom layers are differentiated from the middle layer by the existence of abundant saline crystals and large to medium nahcolite (NaHCO3) crystals that, in many cases, have been voided through dissolution increasing the secondary porosity. Outcrop studies have shown numerous faults and fissures. Thus, this generally tight aquifer has increased secondary permeability and porosity. Core studies have suggested that hydraulic conductivity might be higher at locations 14X-34, 14-36, X-13, 17, 4, and Corehole 2, due to significant to extensive dissolution of the nahcolite and saline minerals. Location 42-34 might have high conductivity due to several minor thin silty beds that could increase permeability. Corehole 9, Skyline 16, EX-1, and Red Wash 1 show 
only partial dissolution that might or might not significantly increase conductivity. Utah State 1 has no signs of dissolution [29]. Drawdown tests on three observation wells produced a 0.08 feet/day to 118.1 feet/day range of average horizontal conductivity [30].

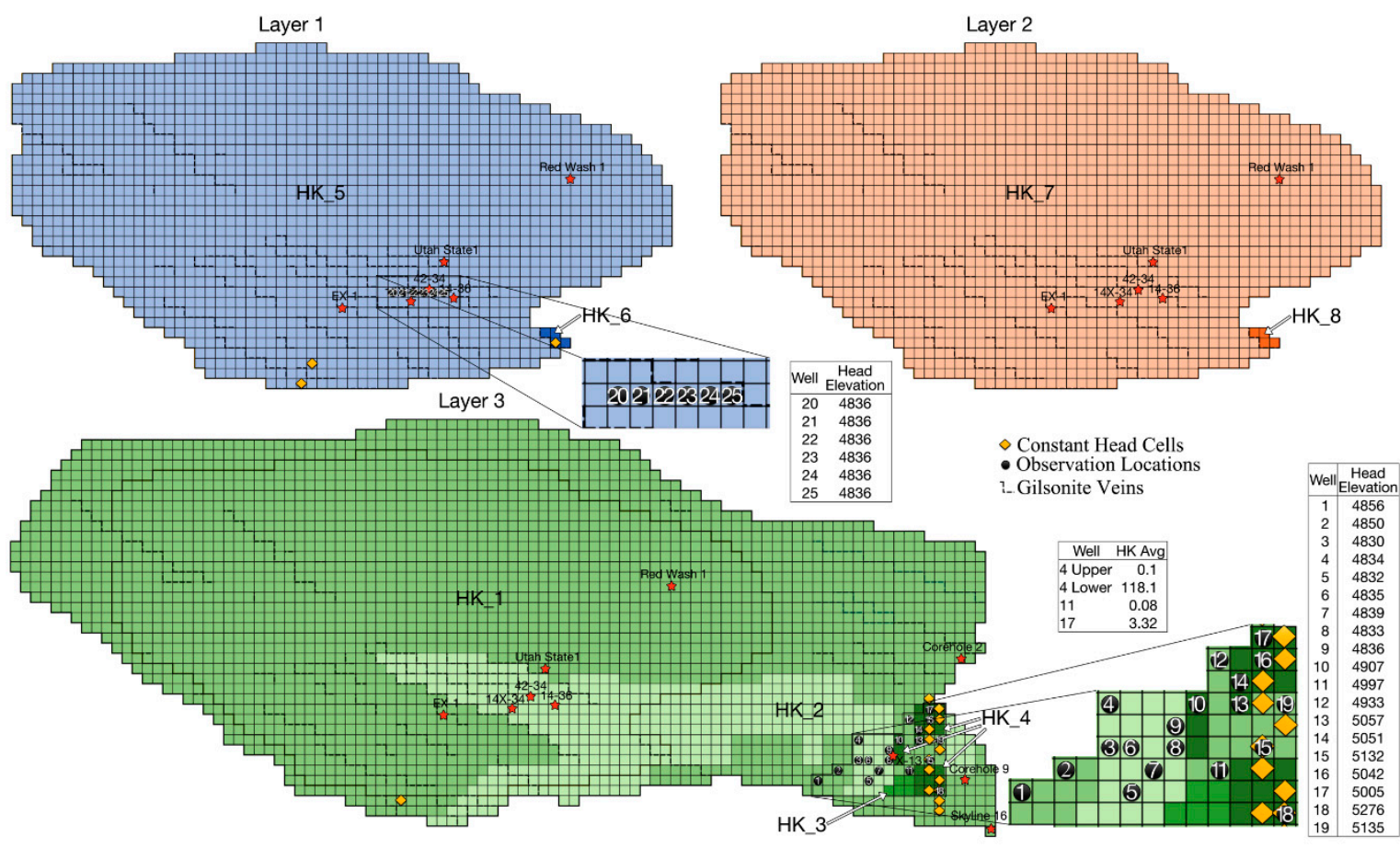

Figure 2. Three-layer Birds Nest Aquifer (BNA) model constant head cells, observation locations, layers, and horizontal conductivity zones (indicated by different shades and HK_\#). Inset closeups of observation well locations and adjacent tables of observed head elevations. Inset table of average conductivity estimates from well drawdown tests.

BNA is represented in MODFLOW as a three-layer 3-D model with 42 rows and 97 columns. Each cell is a half mile long per side. Seventeen constant head cells simulate discharge and recharge. There are 25 head observation locations. All layers are confined and vertical conductivity is proportional to horizontal conductivity. The current BNA model has eight horizontal hydraulic conductivity zones, designated by HK_\#.

This paper focuses on calibrating the horizontal hydraulic conductivities for the eight zones existing in the current BNA model. Based upon data from core studies and drawdown tests, our first draft BNA model had about 50 horizontal conductivity zones. Further guided by that data and preliminary calibration runs, we combined most zones that had similar conductivities. The range of horizontal conductivities reported from pump tests serve as the upper and lower bounds on values considered during the calibration runs reported here.

Our calibration comparison employed 15 runs per combination of optimizer and number of agents (preliminary 15 run-trials per optimizer captured a wide range of results for each optimizer). Each combination run performed approximately 9660 MODFLOW simulations. The number of agents (size of the agent pool) for each run was either 30 or 100. There is no consensus on how many agents to use for an optimizer. For this effort, 30 and 100 agents give an adequate range of results. The five-character name assigned to a combination consists of the three-digit optimizer abbreviation, followed by the number of agents.

\section{Results}

Table 2 shows that for 15 runs of the tested methods, GWO produced the hydraulic conductivity value sets that yielded the best simulated head-best (lowest) root mean squared error (RMSE), lowest 
average RMSE, and narrowest range of RMSEs. A narrow RMSE range indicates that there was little difference in RMSE produced by each run. As shown later, the GWO runs also produced small ranges in values of each of the hydraulic conductivity values. In this case, it might be possible to use any single GWO optimizer run, knowing that its results will not be significantly different than any other GWO run, including outliers. Whereas, if using a PSO optimizer, it would be wise to make multiple optimizer runs because the conductivity values estimated by different runs can be statistically significantly different. PEST matched GWO for the best run, but the PEST average RMSE and range were significantly larger than all global optimizers, by an order of magnitude.

Table 2. Average and minimum (best) results from 15 runs from each pairing of optimizer and number of agents (best result in bold green, second best in orange).

\begin{tabular}{|c|c|c|c|c|c|c|c|}
\hline Variables & DRB100 & DRB30 & PSO100 & PSO30 & GW0100 & GWO30 & PEST \\
\hline Time (s) Avg & 2955 & 3005 & 3082 & 3197 & 3010 & 3053 & 114 \\
\hline Time (s) Min & 2918 & 2955 & 2066 & 2507 & 1768 & 1762 & 12 \\
\hline RMSE (ft/d) Avg & 5.61 & 5.39 & 9.14 & 8.93 & 4.41 & 4.25 & 88.82 \\
\hline RMSE (ft/d) Min & 5.03 & 4.59 & 5.52 & 5.18 & 4.19 & 4.19 & 4.19 \\
\hline$\sigma$ & 0.29 & 0.53 & 3.36 & 2.04 & 0.14 & 0.04 & 20.43 \\
\hline Range (ft/d) & 1.15 & 1.90 & 11.60 & 8.48 & 0.50 & 0.12 & 186.55 \\
\hline $\begin{array}{l}\text { Iteration no. of last } \\
\text { RMSE Improvement }\end{array}$ & 8390 & 8068 & 5537 & 4943 & 9569 & 9618 & 333 \\
\hline
\end{tabular}

The average processing times of the different global optimizer runs in Python were very similar. However, PEST, a local optimizer, required many fewer MODFLOW simulations than the global optimizers. The Figure 3 plots of RMSE improvement with iteration for the best run of each method, shows that initially, PEST obtained better results in fewer simulations, followed by DRB with 100 agents and then PSO and GWO with 30 agents. However, as the number of simulations increased, GWO matched the best final RMSE of PEST.

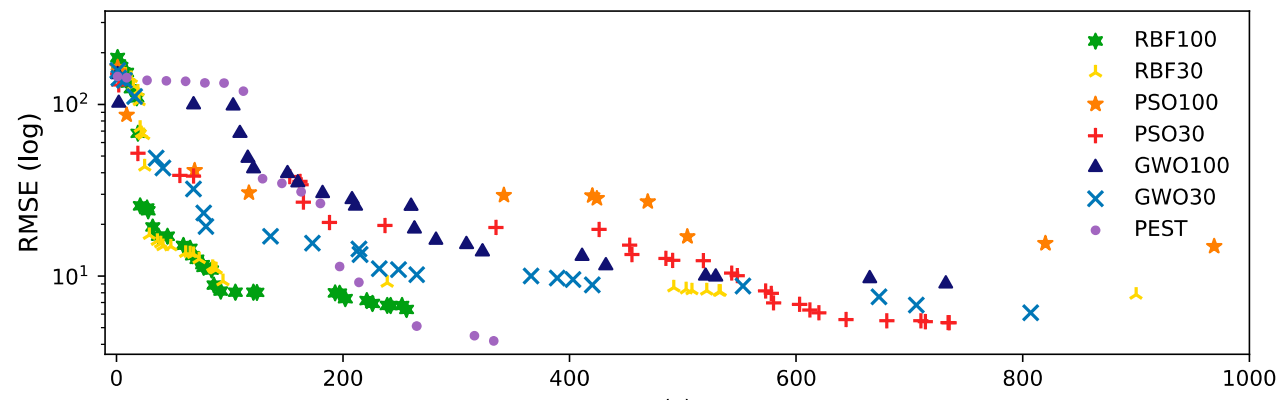

(a)

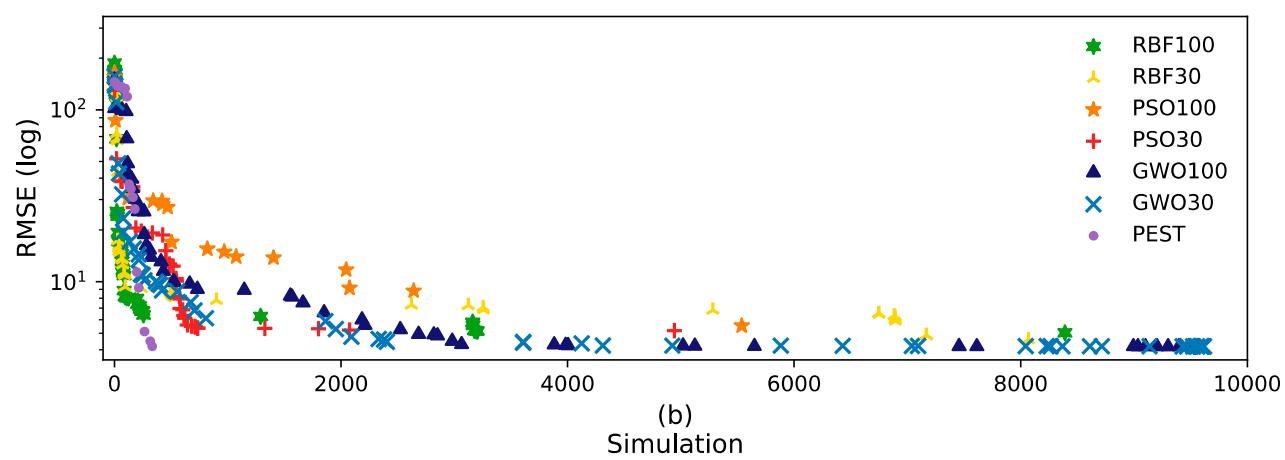

Figure 3. RMSE improvements with simulation of the best run of each method-(a) 1000 simulations and (b) 10,000 simulations. 
The average optimizer performance, shown in Figure 4, highlights that in DRB and GWO, the number of agents does not change the results much. Generally, DRB appears to quickly resolve to its optimum and then, on average, produces no better fitness over time. However, the running improvements of best run shows that DRB fitness might slowly improve. GWO resolves nearly as quickly as DRB, but continues to improve continuously, resulting in a better final fitness. Initially, PSO with 30 agents performs similarly to GWO, but does not resolve to as good a fitness. Overall, performance of PSO lags behind that of DRB and GWO.

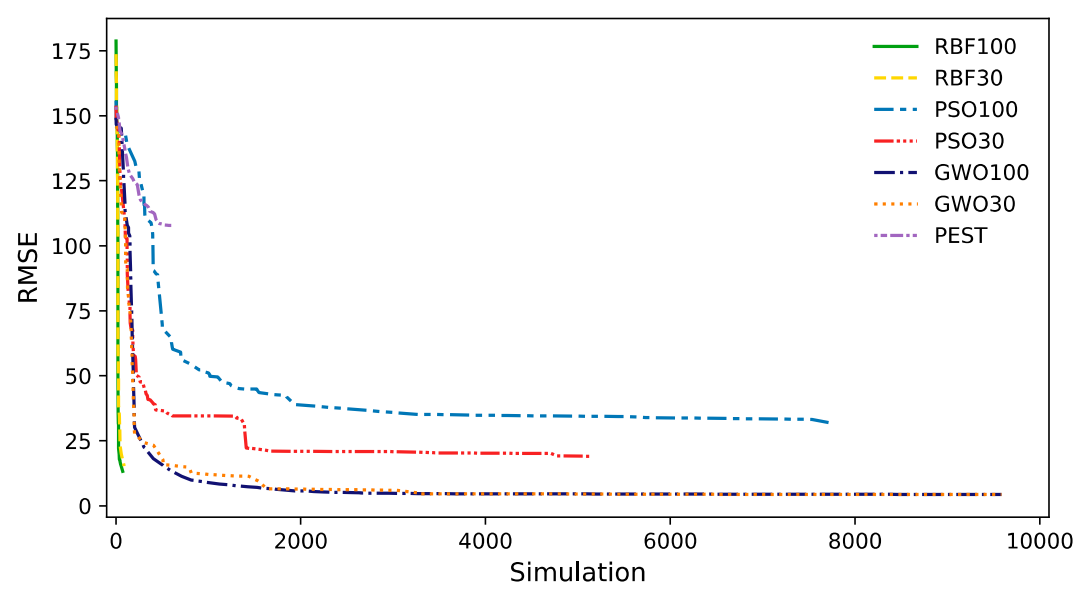

Figure 4. Running improvements of the mean of 15 runs by optimizer/agent size pairing.

PEST matched the best RMSE in one run, and did so much more rapidly, than any other method, but the average PEST run performance is worse than any of the global optimizers. Nevertheless, PEST ran an average of merely 278 MODFLOW simulations in 15 runs, and more runs might have yielded better results (The results of an extra 15 random runs in PEST confirmed that the first 15 runs had explored the search space effectively, without getting a better solution). All the global optimizers ran about 10,000 simulations, but only GWO continuously improved throughout the entire set of simulations. PSO's RMSE was about double of that of GWO and only continued to improve from about half to three-fourths of the total number of simulations. DRB appears to be the most efficient of the global optimizers, because it achieved a low RMSE in relatively few simulations, with a low average RMSE that was better than PSO. However, GWO consistently produced the lowest RMSE.

The Figure 5 boxplots display the range and average of RMSE results from tested optimizer combinations and PEST. The GWO30 combination provided the lowest mean, $4.25 \mathrm{ft} / \mathrm{d}$, and the smallest range, $0.12 \mathrm{ft} / \mathrm{d}$, of fitness results and had a low standard deviation of 0.04 . The results from DRB100 were quite good as well with a mean of $5.61 \mathrm{ft} / \mathrm{d}$, a range of $1.15 \mathrm{ft} / \mathrm{d}$, and standard deviation of 0.29. Results from the best PSO option, PSO30, had a mean double of that of either GWO result, and a range about 70 times larger than GWO30 and over 7 times larger than DRB100. PEST exhibits, by far, the largest range and the highest mean. PEST can perform well, but is not as consistent as the global optimizers. 


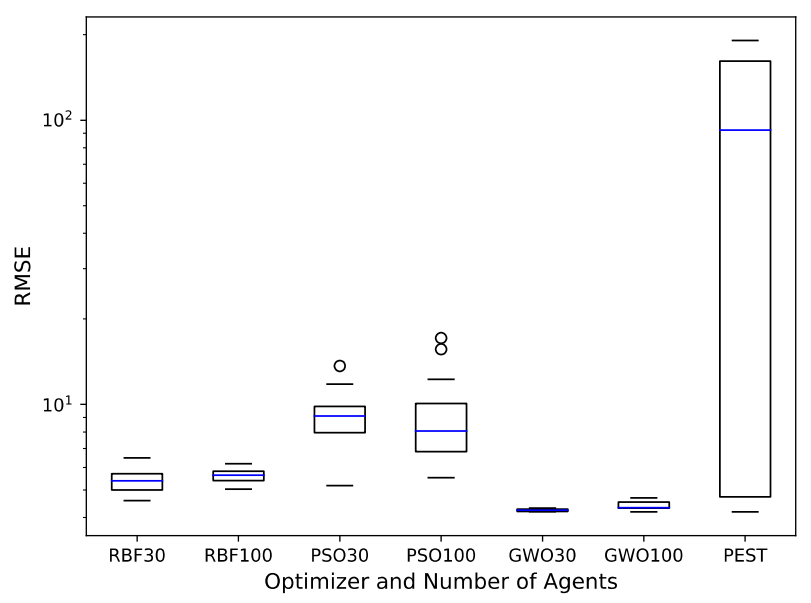

Figure 5. Box plot of the root mean squared error (RMSE) produced by 15 runs of each optimizer and PEST.

Table 3 shows the horizontal conductivity (HK) values from the best runs. For all 15 runs, Table 4 shows the HK standard deviation of each zone. Notably, the values for both GWO and DRB are very small in zones HK_2, HK_3, and HK_4. Zone conductivity standard deviations from PSO and PEST do not vary much in magnitude. All conductivity values presented by PEST and the optimizers were within the imposed upper and lower bounds that we derived from field studies [30]. In fact the low conductivity zone agrees with a previous work modeling study [28]. Our reported HK values, potentiometric surface map, and resulting flow directions are reasonable with respect to previous BNA reports and core studies [28,30]. The HK_1 conductivity reported by PEST, and the HK_5 conductivity reported by PSO30 were at the posed upper bounds. GWO, PSO, and DRB do not compute or report a sensitivity analysis. PEST reports sensitivities that identify parameter changes that most significantly change the resulting RMSE. PEST reports normalized sensitivities for each iteration and composite sensitivities for the entire optimization process. PEST normalizes by dividing each sensitivity value by the number of model observations that it is trying to match.

Table 3. RMSE and horizontal conductivity (HK_\#) values for the best run of each optimizer/agent number pair. Similar values per HK zone are colored the same.

\begin{tabular}{cccccccccc}
\hline & RMSE & HK_1 & HK_2 & HK_3 & HK_4 & HK_5 & HK_6 & HK_7 & HK_8 \\
\hline DRB100 & 5.03 & 37.97 & 0.05 & 0.41 & 0.11 & 78.69 & 60.64 & 61.01 & 49.33 \\
DRB30 & 4.59 & 69.08 & 0.06 & 0.44 & 0.14 & 74.70 & 12.15 & 17.77 & 0.34 \\
PSO100 & 5.52 & 97.31 & 0.02 & 50.63 & 0.62 & 88.09 & 116.36 & 8.52 & 42.07 \\
PSO30 & 5.18 & 33.90 & 0.05 & 0.32 & 0.11 & 118.00 & 66.45 & 45.38 & 92.64 \\
GWO100 & 4.19 & 86.96 & 0.06 & 0.45 & 0.14 & 45.94 & 6.88 & 0.02 & 0.19 \\
GWO30 & 4.19 & 117.73 & 0.06 & 0.45 & 0.15 & 1.78 & 2.13 & 11.08 & 0.20 \\
PEST & 4.19 & 118.00 & 0.06 & 0.45 & 0.14 & 0.32 & 0.83 & 11.27 & 0.22 \\
\hline Upper Bound & & 118.00 & 118.00 & 118.00 & 118.00 & 118.00 & 118.00 & 118.00 & 118.00 \\
Lower Bound & & 0.01 & 0.01 & 0.01 & 0.01 & 0.01 & 0.01 & 0.01 & 0.01 \\
\hline
\end{tabular}

Table 4. Standard deviation of 15 runs of each HK zone by optimizer/agent number pair.

\begin{tabular}{ccccccccc}
\hline & HK_1 & HK_2 & HK_3 & HK_4 & HK_5 & HK_6 & HK_7 & HK_8 \\
\hline DRB100 & 18.27 & 0.017 & 0.30 & 0.067 & 32.90 & 35.80 & 19.03 & 18.88 \\
DRB30 & 18.73 & 0.023 & 0.24 & 0.11 & 28.86 & 37.87 & 34.73 & 30.93 \\
PSO100 & 21.89 & 21.05 & 29.30 & 43.75 & 37.04 & 35.73 & 26.13 & 37.06 \\
PSO30 & 31.38 & 12.86 & 39.05 & 37.54 & 39.36 & 39.57 & 33.49 & 32.66 \\
GWO100 & 27.21 & 0.0010 & 0.007 & 0.0030 & 39.39 & 5.53 & 32.66 & 2.50 \\
GWO30 & 0.21 & 0.0010 & 0.011 & 0.0022 & 19.14 & 3.44 & 32.18 & 3.70 \\
PEST & 47.69 & 24.78 & 41.70 & 25.00 & 39.17 & 21.45 & 46.65 & 34.45 \\
\hline
\end{tabular}




\section{Conclusions}

We compared the results from three Python-based optimizer packages-Grey-Wolf (GWO), particle swarm (PSO), and DYCORS/radial basis function (DRB) - and PEST, when calibrating for eight horizontal conductivity values of the three-layer Birds Nest Aquifer. In terms of computed root mean squared error (RMSE) of simulated head, both GWO and DRB performed as well as, or better, than PEST. Preparation of input data and running GWO is simple. Although DRB has more options requiring input, all preset default options worked well.

Overall, GWO provided the best RMSE, regardless of whether using 30 or 100 agents. Although 100 agents performed better in early simulations, as simulations continued, the results from GWO using 30 and 100 agents became similar. The RMSE values computed by 15 GWO/30 agent runs have the narrowest range of any tested method. The second and third narrowest ranges came from GWO/100 agents and DRB/100 agents, respectively. The narrow range in RMSE results adds confidence that any single GWO optimization would produce good results. Even more important, the set of conductivity values determined by GWO/30 agents are very similar to those by the best PEST results. The range of RMSE results from PEST runs is much larger than any of the global optimizers. Although for this site, PEST did match the best fitness provided by any of the global optimizers, for another more nonlinear calibration problem, a PEST user might be uncertain that they had obtained close to a globally optimal solution.

Our work highlights the promise of the recently developed GWO nature-based optimizer in terms of simplicity of implementation and goodness of results. Further investigations using GWO might promote its use in more fields and applications. It might also be valuable to perform similar comparisons for more complex scenarios that have a much greater dimensionality or more complex interaction between parameters. That additional comparison could test the suitability and the limits of each optimizer.

Author Contributions: Conceptualization, R.T.L. and P.M.; Formal analysis, R.T.L.; Investigation, R.T.L.; Methodology, R.T.L. and R.C.P.; Project administration, R.C.P.; Software, R.T.L. and P.M.; Supervision, R.C.P.; Validation, R.C.P.; Visualization, R.T.L.; Writing-original draft, R.T.L.; Writing-review and editing, R.T.L. and R.C.P. All authors have read and agreed to the published version of the manuscript.

Acknowledgments: We are grateful for the data provided by John Baza, the Utah Division of Oil, Gas and Mining, the Utah Division of Natural Resources. This research was supported by the Utah Water Resources Research Lab, and by the Utah Agricultural Experiment Station, Utah State University, and approved as journal paper number 4110 .

Conflicts of Interest: The authors declare no conflict of interest.

\section{References}

1. Skahill, B.E.; Baggett, J.S.; Frankenstein, S.; Downer, C.W. More efficient PEST compatible model independent model calibration. Environ. Model. Softw. 2009, 24, 517-529. [CrossRef]

2. Mbonimpa, E.G.; Gautam, S.; Lai, L.; Kumar, S.; Bonta, J.V.; Wang, X.; Rafique, R. Combined PEST and Trial-Error approach to improve APEX calibration. Comput. Electron. Agric. 2015, 114, 296-303. [CrossRef]

3. Waterloo Hydrologic Visual MODFLOW Flex; Waterloo Hydrogeologic: Waterloo, ON, Canada, 2019.

4. Rumbaugh, J.O.; Rumbaugh, D.B. Groundwater Vistas; Environmental Simulations, Inc.: Leesport, PA, USA, 2017.

5. Chiang, E. Processing Modflow 10.0.22: A Graphical User Interface for MODFLOW, MODPATH, MT3D, PEST, SEAWAT, and ZoneBudget; Simcore Software: Irvine, CA, USA, 2019.

6. Aquaveo, L.L.C. Groundwater Modeling System Version 10.0.14 (64-bit), Build Date, Mar 4, 2016; Aquaveo, L.L.C.: Provo, UT, USA, 2016.

7. Solomatine, D.P.; Dibike, Y.B.; Kukuric, N. Automatic calibration of groundwater models using global optimization techniques. Hydrol. Sci. J. 1999, 44, 879-894. [CrossRef] 
8. Mugunthan, P.; Shoemaker, C.A.; Regis, R.G. Comparison of function approximation, heuristic, and derivative-based methods for automatic calibration of computationally expensive groundwater bioremediation models: COMPARISON OF OPTIMIZATION METHODS. Water Resour. Res. 2005, 41. [CrossRef]

9. Skahill, B.E.; Doherty, J. Efficient accommodation of local minima in watershed model calibration. J. Hydrol. 2006, 329, 122-139. [CrossRef]

10. Blasone, R.-S.; Madsen, H.; Rosbjerg, D. Parameter estimation in distributed hydrological modelling: Comparison of global and local optimisation techniques. Nord. Hydrol. 2007, 38, 451. [CrossRef]

11. Ng, T.L.; Eheart, J.W.; Cai, X.M. Comparative Calibration of a Complex Hydrologic Model by Stochastic Methods GLUE and PEST. Trans. ASABE 2010, 53, 1773-1786. [CrossRef]

12. Mirjalili, S.; Mirjalili, S.M.; Lewis, A. Grey Wolf Optimizer. Adv. Eng. Softw. 2014, 69, 46-61. [CrossRef]

13. Franssen, H.J.H.; Kinzelbach, W. Ensemble Kalman filtering versus sequential self-calibration for inverse modelling of dynamic groundwater flow systems. J. Hydrol. 2009, 365, 261-274. [CrossRef]

14. Yoon, H.; Hart, D.B.; McKenna, S.A. Parameter estimation and predictive uncertainty in stochastic inverse modeling of groundwater flow: Comparing null-space Monte Carlo and multiple starting point methods: NULL-SPACE MONTE CARLO AND MULTIPLE STARTING POINT. Water Resour. Res. 2013, 49, 536-553. [CrossRef]

15. Haddad, O.B.; Tabari, M.M.R.; Fallah-Mehdipour, E.; Mariño, M.A. Groundwater Model Calibration by Meta-Heuristic Algorithms. Water Resour. Manag. 2013, 27, 2515-2529. [CrossRef]

16. Thomas, A.; Eldho, T.I.; Rastogi, A.K.; Majumder, P. A comparative study in aquifer parameter estimation using MFree point collocation method with evolutionary algorithms. J. Hydroinf. 2019, 21, 455-473. [CrossRef]

17. Majumder, P.; Eldho, T.I. Artificial Neural Network and Grey Wolf Optimizer Based Surrogate Simulation-Optimization Model for Groundwater Remediation. Water Resour. Manag. 2020. [CrossRef]

18. Kennedy, J.; Eberhart, R. Particle swarm optimization. In Proceedings of the Proceedings of ICNN'95-International Conference on Neural Networks; IEEE: Perth, WA, Australia; p. 1995.

19. Matott, L.S.; Rabideau, A.J.; Craig, J.R. Pump-and-treat optimization using analytic element method flow models. Adv. Water Resour. 2006, 29, 760-775. [CrossRef]

20. Mategaonkar, M.; Eldho, T.I. Groundwater remediation optimization using a point collocation method and particle swarm optimization. Environ. Model. Softw. 2012, 32, 37-48. [CrossRef]

21. Gaur, S.; Chahar, B.R.; Graillot, D. Analytic elements method and particle swarm optimization based simulation-optimization model for groundwater management. J. Hydrol. 2011, 402, 217-227. [CrossRef]

22. Faris, H.; Aljarah, I.; Al-Betar, M.A.; Mirjalili, S. Grey wolf optimizer: A review of recent variants and applications. Neural Comput. Appl. 2018, 30, 413-435. [CrossRef]

23. Eriksson, D.; Bindel, D.; Shoemaker, C.A. pySOT and POAP: An event-driven asynchronous framework for surrogate optimization. arXiv 2019, arXiv:1908.00420.

24. Regis, R.G.; Shoemaker, C.A. A Stochastic Radial Basis Function Method for the Global Optimization of Expensive Functions. Inf. J. Comput. 2007, 19, 497-509. [CrossRef]

25. Regis, R.G.; Shoemaker, C.A. Combining radial basis function surrogates and dynamic coordinate search in high-dimensional expensive black-box optimization. Eng. Optim. 2013, 45, 529-555. [CrossRef]

26. Pyswarm. Available online: https://github.com/tisimst/pyswarm (accessed on 28 June 2019).

27. SwarmPackagePy. Available online: https:/github.com/SISDevelop/SwarmPackagePy (accessed on 28 June 2019).

28. Holmes, W.F.; Kimball, B.A. Ground Water in the Southeastern Uinta Basin, Utah and Colorado, U.S. Geological Survey Water-Supply Paper 2248; U.S. Geological Survey: Reston, VA, USA, 1983; p. 164.

29. Vanden Berg, M.D.; Lehle, D.R.; Carney, S.M.; Morgan, C. Geological Characterization of the Birds Nest Aquifer, Unita Basin, Utah: Assessment of the Aquifer's Potential as a Saline Water Disposal Zone; Utah Geological Survey: Salt Lake City, UT, USA, 2013; ISBN 978-1-55791-874-1.

30. Final Environmental Baseline Report: Federal Prototype Oil Shale Leasing Program Tracts UA and UB. Utah: White River Shale Project; VTN Colorado, Inc.: Aurora, CO, USA, 1977.

(C) 2020 by the authors. Licensee MDPI, Basel, Switzerland. This article is an open access article distributed under the terms and conditions of the Creative Commons Attribution (CC BY) license (http://creativecommons.org/licenses/by/4.0/). 